$9-1-2020$

\title{
Improving reproductive health and family planning outcomes among out-of-school adolescents and youth in the Philippines
}

Breakthrough RESEARCH

De La Salle University

Follow this and additional works at: https://knowledgecommons.popcouncil.org/departments_sbsr-pgy

Part of the Health Communication Commons, and the Public Health Commons How does access to this work benefit you? Let us know!

\section{Recommended Citation}

Breakthrough RESEARCH and De La Salle University. 2020. "Improving reproductive health and family planning outcomes among out-of-school adolescents and youth in the Philippines," Programmatic Research Brief. Washington, DC and Manila: De La Salle University and Population Council. 


\section{Improving Reproductive Health and Family Planning Outcomes Among Out-of-School Adolescents and Youth in the Philippines}

Across the globe, studies suggest that schooling is one of the most important factors shown to lead to positive reproductive health $(\mathrm{RH})$ outcomes. ${ }^{1}$ Evidence suggests that young people who do not attend school are more sexually active than their in-school counterparts and engage in more sexual risk behaviors. ${ }^{2,3}$ School-based programs that educate young adults about their health are proving effective ${ }^{4}$, but such programs exclude young people not attending school. ${ }^{5}$

In the Philippines where adolescent and youth reproductive health (AYRH) is of considerable concern, youth have limited knowledge and sources of information about their reproductive health. In the 2013 Young Adult Fertility and Sexuality study, only 27 percent of Filipino youth reported believing that they have sufficient knowledge about sex, and only 15 percent could correctly identify when conception is most likely to occur during a woman's menstrual cycle. Further, the Philippine adolescent fertility rate (AFR) ranks second highest in Southeast Asia ${ }^{6}$ and the Philippines was the only country in Southeast Asia with an AFR that increased from 2011 to 2014.

Although parents are considered the primary source of knowledge and values about sex in the Philippines, the proportion of Filipino youth who ever discussed sex at home was less than 10 percent, with 22 percent of youth reporting that they chose not to discuss these topics with anyone. ${ }^{7}$ Filipino youth reportedly have low exposure to sexual and reproductive health-related educational materials and information. While there are gender differences in sexual behaviors and knowledge among Filipino youth, educational attainment plays an important role: less-educated youth consistently exhibit more risky sexual behaviors and possess less knowledge about sex and reproduction, and little is known about the sexual and reproductive health-related knowledge, behaviors, and needs of out-of-school youth (OSY) (see Box 1 for definition), a particularly vulnerable group.

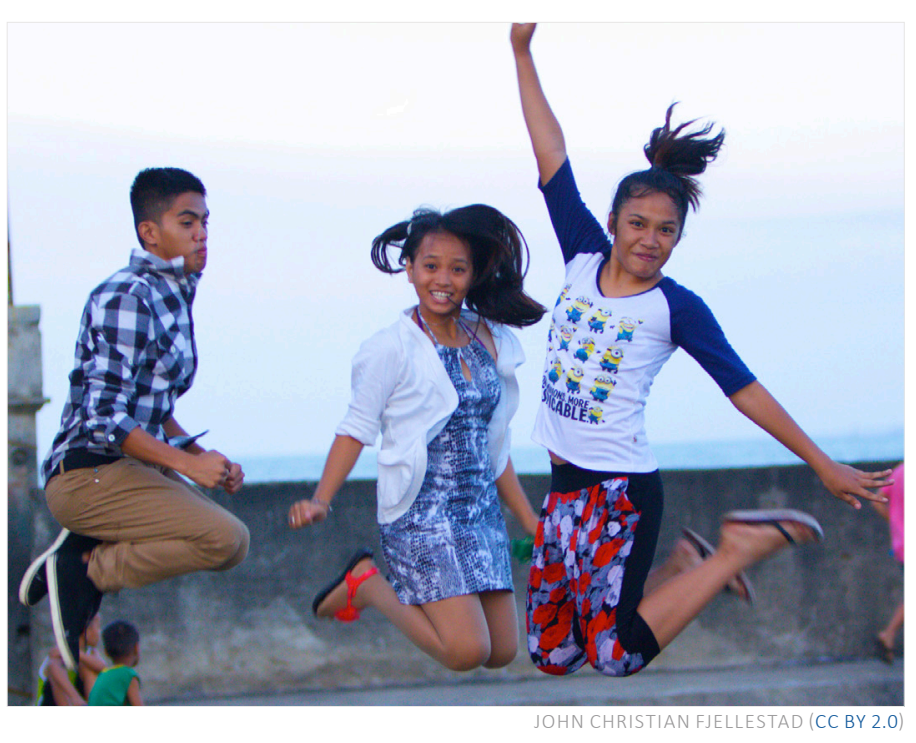

Most OSY are dependent on their in-school peers and mass media for RH information. These sources, however, also can provide misleading or incorrect information, which can lead to decisions that can negatively affect AYRH. ${ }^{8}$ In the Philippines, interventions to increase knowledge are needed, especially as the country faces a large OSY population, with school withdrawals primarily due to family poverty. ${ }^{9,10}$ OSY require more focused interventions than their school-attending counterparts. ${ }^{11}$ One approach to reach OSY is social and behavior change (SBC) programming directed to fill critical information gaps to help reduce the risk behavior and improve AYRH outcomes.

The Population Council, through Breakthrough RESEARCH, supported with funding from United States Agency for International Development (USAID) Philippines, and in collaboration with De La Salle University's Social Development Research Center (DLSU-SDRC) in Manila, conducted a targeted qualitative research study to generate evidence to facilitate SBC approaches to reach OSY ages 15 to 19 to affect positive reproductive health and family planning behavior change among adolescents in three regions in the 


\section{BOX 1 WHO IS AN OUT-OF-SCHOOL YOUTH?}

For this study, an OSY is an individual between the ages of 15 and 19 who is not currently enrolled in any school or formal education provider. He or she may be out of school for any reason, e.g., work, illness, lack of finances, or pregnancy, among other reasons.

In the Philippines, several governmental and nongovernmental agencies and organizations are involved in various programs and services for adolescents and youth. The age range of beneficiaries, however, significantly varies depending on the implementing agency or organization. For the Department of Social Welfare and Development (DSWD) and the Population Commission, 15- to 24-year-olds are referred to as youth. The Department of Education (DepEd), however, adopts a wider age range, defining "youth" as people between the ages of 7 and 30 years old. This has some overlap with the World Health Organization (WHO) definition of adolescents (10 to 19 years old) and the United Nations definition of youth (15 to 24 years old) and young people (10 to 24 years old). In the 2013 Functional Literacy, Education, and Mass Media Survey (FLEMMS), the Philippine Statistics Authority (PSA) defined OSY as persons aged 15 to 24 years old who are not attending school, have not finished any college or post-secondary course, and are not working.

Philippines: Manila and Quezon City in the National Capital Region, Cebu City in the Central Visayas Region, and Davao City in the Mindanao Region.

\section{Study Objectives}

This study aimed to generate evidence for the design of intervention approaches most effective in reaching OSY between 15 and 19 years old in the Philippines, for four priority FP and RH behaviors:

1. Delay of sexual debut.

2. Use of modern contraceptive methods to prevent pregnancy.

3. Care-seeking in the first trimester for pregnant youth.

4. Birth spacing of three to five years to prevent short birth intervals.

The study's key objectives are to:

- Document the reproductive health and family planning behaviors of OSY 15-19 years of age.

- Identify which channels and influencers are most effective in reaching OSY ages 15 to 19.

- Generate evidence for message framing that will resonate with OSY.

- Provide recommendations and guidance for RH/FP programming targeted toward OSY.

\section{Methodology}

The study was conducted in four major cities in the Philippines: Manila, Quezon City, Cebu, and Davao. The people of Manila and Quezon City speak Filipino, the cities' vernacular, while those in Cebu and Davao speak the Visayan language. All four cities' citizens are conversant in English as a second language. This study was designed as a qualitative research study and utilized three qualitative research methodologies: 1) focus group discussions (FGDs), 2) in-depth interviews (IDIs), and 3) key informant interviews (KIIs). Researchers from DLSU-SDRC led the implementation of the study on the ground in the Philippines, including fieldwork training, data collection, transcription, translation, and analysis, in close consultation with the Population Council.

Trained facilitators and interviewers conducted research activities among OSY, their parents, and key informants to gather information on FP and RH knowledge, attitudes, and practices with a focus on the four priority behaviors of interest. To ensure that participants fully understood the questions asked, the tools were translated into the vernacular of each site (Tagalog for the Manila and Quezon City participants and Bisaya for Cebu and Davao participants).

While FGDs collected normative information from OSY participants, i.e., experiences of OSY of the same age but not their own personal experiences, IDIs explored various $\mathrm{FP}$ and $\mathrm{RH}$ experiences of the individual OSY interviewed. FGDs were conducted among different groups of OSY based on their age (15 to 17 years old or 18 to 19 years 
TABLE 1 DATA COLLECTION METHODS

\begin{tabular}{|c|c|c|c|}
\hline & 1. Focus group discussions (FGDs) & 2. In-depth interviews (IDIs) & 3. Key informant interviews (KIls) \\
\hline Study population & $\begin{array}{l}\text { Out-of-school youth (OSY): } \\
\text { Enrolled in adolescent programs } \\
\text { Identified in the communities/ } \\
\text { barangays/local government units } \\
\text { Living on the street } \\
15-19 \text { years old } \\
\text { Male or female } \\
\text { Cohabiting or single } \\
\text { Mothers/fathers/guardians of OSY } \\
\text { enrolled in programs or living in } \\
\text { communities or on the street. }\end{array}$ & $\begin{array}{l}\text { A subset of OSY who } \\
\text { participated in FGDs }\end{array}$ & $\begin{array}{l}\text { Local stakeholders in Quezon City, } \\
\text { Manila, Davao, and Cebu } \\
\text { National stakeholders in Manila }\end{array}$ \\
\hline Sample size & $\begin{array}{l}\text { Per study site (4 study sites): } \\
8 \text { FGDs with OSY } \\
2 \text { FGDs with parents } \\
\text { Total: } 40 \text { FGDs }\end{array}$ & $\begin{array}{l}\text { Per study site (4 study sites): } \\
4 \text { IDIs } \\
\text { Total: } 16 \text { IDIs }\end{array}$ & $\begin{array}{l}\text { Local: } 22 \text { KIIs ( } 5 \text { in Quezon City } \\
\text { and Manila, } 6 \text { in Davao and Cebu) } \\
\text { National: } 8 \text { KIls }+1 \text { key influencer } \\
\text { Total: } 31 \text { KIls }\end{array}$ \\
\hline $\begin{array}{l}\text { Location of } \\
\text { activity }\end{array}$ & $\begin{array}{l}\text { Manila } \\
\text { Quezon City } \\
\text { Davao } \\
\text { Cebu }\end{array}$ & $\begin{array}{l}\text { Manila } \\
\text { Quezon City } \\
\text { Davao } \\
\text { Cebu }\end{array}$ & $\begin{array}{l}\text { Manila } \\
\text { Quezon City } \\
\text { Davao } \\
\text { Cebu }\end{array}$ \\
\hline Timing & June 2019-July 2019 & June 2019-July 2019 & June 2019-July 2019 \\
\hline Method & FGDs & IDIs & Semi-structured interviews \\
\hline
\end{tabular}

old), marital status (cohabiting or non-cohabiting), and gender (male or female). Participants were recruited from OSY-focused institutions, OSY communities, and the streets. IDIs were conducted among a sub-sample of those who participated in the FGDs, selected with consideration of marital status (cohabiting or not), gender (male and female), and age (15 to 19), and featured open-ended questions related to sexual behaviors, RH, and FP. KIIs were conducted as semi-structured interviews among key stakeholders who are knowledgeable about, or work on, FP and RH issues among OSY at nationally and locally in the Philippines. Stakeholders from diverse backgrounds, representing national, local, governmental, nongovernmental (NGO), and civil-society were interviewed on how SBC programming can be made more effective in message framing and reach, and how various stakeholders can work together toward these goals.

Qualitative data analysis and coding using NVIVO software was conducted using transcripts of recorded interviews and group discussions. Transcripts in local dialects were translated into English.

Informed consent for participation (including audio recording) was obtained from all participants. In compliance with ethical standards for research and the Family Code of the Philippines, OSY participants aged 18 to 19 were requested to provide their informed consent prior to FGD participation; similarly, a parental consent form was given to the parents or guardians of unmarried OSY participants aged 15 to 17 prior to their participation. After the study team obtained a signed parental consent form, each respective unmarried OSY participant age 15 to 17 who agreed to participate in the study also was asked to sign an adolescent consent form. The study tools and research protocol underwent rigorous ethical review both with the Population Council Institutional Review Board in New York and the De La Salle University Research Ethics Office in Manila prior to the study's commencement. 


\section{Results}

\section{Priority behaviors of OSY and adolescents}

\section{Early sexual debut}

Both single and cohabiting OSY reported their first sexual experiences as being unplanned. Most reported sexual initiation at a very early age (i.e., between 13 and 16) and that the behavior was largely inevitable. OSY early sex occurred for a number of reasons including sexual attraction, lust, love, or curiosity, and some respondents reported that sex was occasionally in exchange for money. OSY respondents described their sexual encounters and sexual activity from two perspectives: ideal and reality. From an ideal perspective, OSY stated that sex would only take place when both partners are financially ready, having completed schooling, and capable of accepting adult responsibilities. In reality, however, despite awareness of the risks of early sex, including unintended pregnancy and sexually transmitted infections (STIS), OSY believe sexual initiation and encounters at their age are inevitable, in circumstances aided by alcohol or substance abuse in common social situations. Although OSY noted that having a boyfriend or a girlfriend is considered normal, they largely keep their sexual encounters a secret from their parents. Most OSY reported that they spoke about their sexual encounters only with their close friends (barkada/ ka tropa).

\section{For me, doing it [sex] early is not good because they are not thinking about their future, that the child will be the one to suffer because we do not have work and we have nothing to eat.}

-IDI, single male, Quezon City

\begin{abstract}
At our young age, we are not that familiar with the things we do, so I got pregnant. We were not aware that these things [hardships] can happen...the baby is here, we have no choice...if we can turn back the time, we would choose the way not to be pregnant.
\end{abstract}

-IDI, cohabiting female, Manila

Most OSY respondents pointed to the confluence of several factors that signify vulnerabilities they face that lead to early sexual debut. Financial difficulties in the family lead to school withdrawal and risky behaviors, with many from broken homes, lacking parental guidance, or problematic relations or disharmony within the home. OSY noted that regardless of age and gender, early sexual debut results from factors such as their need to feel loved or cared for, curiosity, or stress. Some single male and female OSY respondents did report abstinence out of fear of pregnancy, parental disapproval, or expulsion from school.

\section{The lack of parenting skills in the family is often the underlying reason for children and youth staying away from home and seeking the company of another.}

-KII, program manager of government agency, Manila

\section{Use of modern contraceptive methods to pre- vent pregnancy}

Most OSY respondents in the four study sites, regardless of gender, age, or cohabitation status, exhibited low levels of knowledge, and limited use of contraception despite their sexual activity. Most respondents were under the age of 18 and encounter cultural and logistical barriers to access contraceptives of their choice, as well as youth-friendly contraceptive services that are affordable, confidential, and non-judgmental. When respondents, particularly those cohabiting, reported contraceptive use, the oral pill was the most common method reported among females, while condoms were the most common method reported among males. Some respondents reported a preference for abstinence. As expected, cohabiting females in all sites expressed relatively greater knowledge of contraceptive methods than their non-cohabiting counterparts, particularly if they were already mothers.

Although OSY respondents, both female and male, are aware that contraceptives prevent pregnancy, they lacked further knowledge of specific kinds of contraceptives. Study responses suggest that low contraceptive use among OSY is due to misconceptions about its use, particularly due to what they have heard from others who may have used contraceptives.

\footnotetext{
They say about pills, this is actually has been spread over the Internet, that it does not totally dissolve, that is, it just gets stuck in the ovary. So, what I thought was even if you submerged it in water, it will dissolve in five minutes.
} 
How much more inside your stomach where it could dissolve immediately.

-IDI, cohabiting female, Quezon City

I don't want to use IUD or implant because with my friend's experience, she got pregnant even when she had an IUD.

-IDI, cohabiting female, Manila

\section{Care-seeking during the first trimester of pregnancy}

OSY respondents also generally exhibited low levels of knowledge about the importance of care-seeking during the first trimester of pregnancy. Cohabiting OSY who were already parents were more knowledgeable about care-seeking during the first trimester due to their experience. Single OSY largely had no awareness of the importance of antenatal care (ANC), a finding corroborated by official health and NGO workers.

In terms of care-seeking, generally adolescents more likely do not want to go in health centers anymore. Most of them do not have education so misconceptions arise.

-KII, NGO worker, Manila

OSY who were aware of the importance of ANC deemed care-seeking during the first three months of pregnancy as critical for the health of both the mother and infant, and emphasized the health of the infant as paramount. OSY reported behaviors such as visiting a health facility for ANC as a concrete activity for child health as well as adopting positive prenatal behaviors such as nutritious food, avoiding strenuous activities, and stopping harmful habits such as smoking and drinking. In some cases, a major barrier for seeking appropriate care during the first trimester of pregnancy is that some young women are often unaware of their own pregnancy. Health workers reported that some young women also do not seek care because of hiding their pregnancy from their parents and others due to their fear of social stigma.

Among cohabiting OSY, respondents described seeking care early during pregnancy as a shared responsibility, with male respondents particularly reporting supporting their pregnant partners for seeking care. Single respondents, particularly young women who had given birth, reported seeking advice and support during pregnancy from their mothers especially. Respondents deemed barangay health centers to be reliable and a preferred choice to hospitals, primarily citing their accessibility and affordability.

If you are determined that you are really a husband, you really take care of your wife. If she has someone to go with, if not let's hope nothing happens, hope there's not emergency. You also know the clinic's report about the child coming.

-IDI, cohabiting male

\section{Birth spacing of three to five years to prevent short birth intervals}

OSY respondents in our study evinced the least familiarity with birth spacing and its relevance to RH and FP. Spacing subsequent births after the first child is simply not part of the consciousness of OSY, regardless of age, gender, or cohabitation status. KIls suggest, however, that health providers, NGOs, and others have implemented programs that provide FP counseling with a focus on birth spacing. NGO workers noted that young women do not voluntarily want to get pregnant again after experiencing pregnancy as a teenager, but lack of access to information and services are key barriers putting OSY at elevated risk of short birth intervals.

The case usually is they don't want to have
another baby, but before the year is over,
they will already be pregnant with their next child.
That's because they don't have access, and they're
not given these services. That's why we see that
it's crucial for them to receive antenatal and post-
natal care and services...when they come to the
hospital, you'll advise and provide them with the
IUD, then they'll be protected for the next nine or
five years.

-KII, NGO service provider

\section{Communicating RH and FP messages to OSY}

OSY participants in this study, irrespective of gender, age, and cohabitation, reported obtaining information on FP and $\mathrm{RH}$ behaviors from multiple sources, including interpersonal communication, and social and mass media. They revealed nuanced and specific preferences regarding channels, and message framing. 


\section{Source and channel characteristics}

Social media is the primary source for FH and FP information for OSY: OSY identified the Internet, in particular social media, as their primary source for RH and FP information. In particular, OSY noted using platforms such as Facebook or online messaging or chat groups. Other sources mentioned include YouTube and Wikipedia, friends and peers, and traditional media, such as television and printed media. OSY noted that they search for and watch pornographic videos, music videos, social media memes, and songs on these platforms. Although OSY generally did not report talking about sex with their parents or family members, parents reported that they are typically the first to provide advice or information on these topics, particularly concerning issues of childcare and birth spacing if they have already had their first child. For childcare matters, OSY reported relying on parents as well as health providers due to their perceived trustworthiness and credibility.

OSY prefer sources and channels that are accessible and anonymous: OSY and stakeholders both pointed to social media and the Internet as the most accessible channels to receive information. Additionally, these sources were also deemed to be the most anonymous, enhancing their desirability. Anonymity is a desired characteristic because OSY report preferring to not openly discuss issues related to sex or sexuality with parents, elders, or other members of their community, as they will be judged or stigmatized. OSY participants did not attribute trustworthiness to internet and social media sources specifically despite reporting that it is their primary source for $\mathrm{RH}$ and FP information.

OSY seek information from interpersonal sources that are perceived as trustworthy and relatable: Perceived trustworthiness and relatability of sources of information, which was reported as important by OSY, were largely attributed to interpersonal sources with direct FP and $\mathrm{RH}$ experience. OSY value not only the experience of elders (i.e., parents or older peers), but also the credibility and expertise of professionals (e.g., doctors, nurses, midwives, workers in barangay health centers). Relatability was commonly attributed to peers (i.e., friends), and emphasis on relatability among peers was shared by male and female OSY participants. IDI participants reported seeking information from relatable peers particularly for sexual debut and care-seeking. Relatable peers are also critical for linking OSY with other sources and channels, specifically the social media. OSY noted that for a source to be considered trustworthy it has to be relatable in addition to being credible.

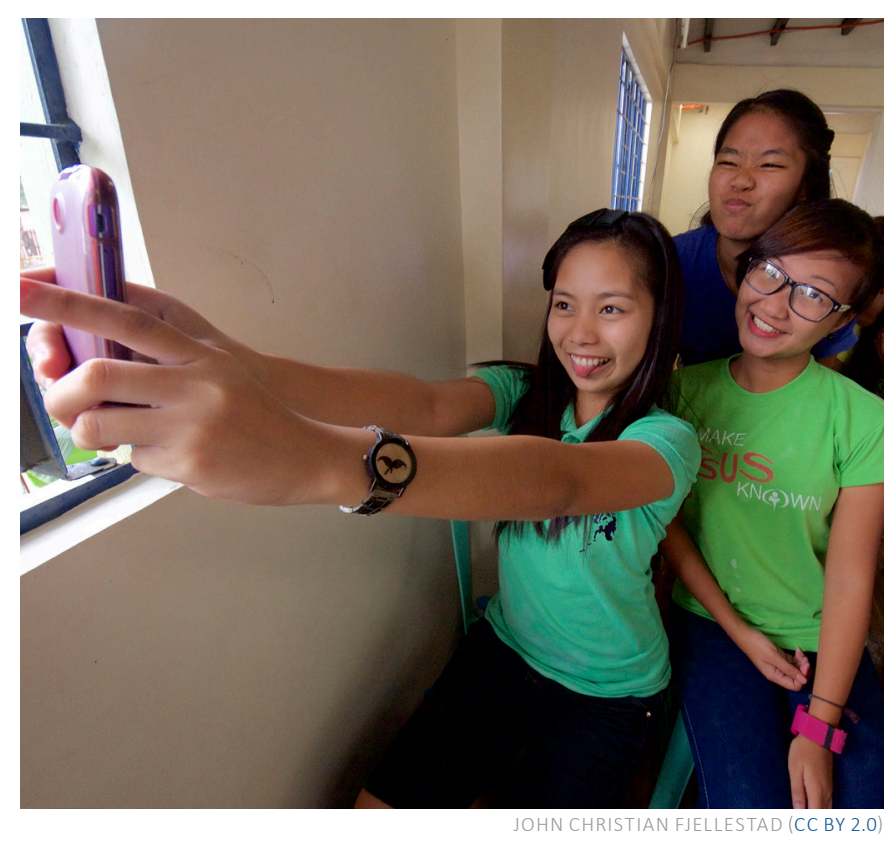

The information is believable if it is from the doctor. Is there any other?

-FGD, 18 to 19 years old, married, male, Quezon City

\section{They [my parents]...know it best because they had already experienced it.}

-FGD, 18 to 19 years old, married, female, Cebu

\section{Message framing}

Adolescents prefer call-to-action messages that are framed as questions: When asked to describe the messages that resonated from various sources, OSY consistently referred to questions structured as calls to action and reported two distinct features of these calls to action that are appealing: 1) messages that encourage OSY to seek advice and further information related to priority $\mathrm{RH}$ and FP behaviors, particularly use of contraceptives; 2) messages that suggest ways to connect with sources of information or services, and assure OSY of their accessibility and anonymity. These messages directed OSY to sources where they could ask questions about any of these priority RH and FP areas. Importantly, these messages evince the availability of preferred information sources for OSY's communication.

\section{Adolescents prefer loss-framed messages that are accompanied by instructions related to addressing risky behaviors: Besides messages that encourage informa- tion-seeking, those that communicated unfavorable consequences of risky behaviors-such as the possibility}


of acquiring STIs-were mentioned as effective by OSY. Unfavorable consequences of risky behaviors are often interpreted within the contexts of present economic difficulties and future goals and ambitions. OSY emphasized the importance of loss-oriented messages accompanied by messages promoting self-efficacy: In addition to negative consequences, messages should include instructions on how to address risky behaviors and their consequences.

\section{They really should not be careless about having sex, because there's a lot of diseases out there these days. That's why they shouldn't be careless about it. That's what they should learn.}

-FGD, 15 to 17 years old, married, female, Davao

\section{Maybe what are the harmful things that could occur when you engage in sex or the do's and don'ts of it?}

-IDI, married, female, Manila

Adolescents appreciate the use of visuals and examples for illustrating the negative consequences of risky behaviors: OSY also indicated the importance of visual images of the negative consequences of risky behaviors. For OSY, visuals may be helpful in illustrating the relationship between risky behaviors and negative consequences. OSY in particular emphasized the utility of STI visuals. KIls also revealed the importance of visuals when communicating with OSY. A barangay health staff member from Davao shared:

The teens here are different, because they seem to lack knowledge, but they respond well to visual media. They will say, 'Oh, right, so that's how it is.' They wouldn't understand the material well, especially when the explanations are in Tagalog, or in deep Binisaya. They wouldn't listen to those. For them, when they see something visual, they say, 'Ah, so that's how it is. That's wrong.' Visual media is effective.

-IDI, barangay health staff member, Davao

\section{Factors that influence OSY RH and FP behaviors}

Findings from this study suggest that interpersonal interactions with credible and caring family members (e.g., parents) and friends or peers, as well as with reproductive health professionals and media personalities that the youth identify with influence OSY RH and FP behaviors across all four priority behaviors (sexual debut, use of contraceptives, care in the first trimester, and birth spacing).

Our findings suggest:

a. Peers who have experienced sexual intimacy influence OSY sexual behaviors.

b. Peer influence becomes deleterious when self-control and regulation are compromised among OSY, such as when substance abuse is the norm among youth groups and barkadas; drinking sprees are often environments for sexually intimate encounters among Filipino youth.

c. Interactions with credible persons (such as medical professionals) outside immediate circles of family and friends were also cited as a factor influencing Filipino OSY behaviors.

A second set of factors that influence OSY behaviors include interactions with traditional media and the influence of media personalities and influencers:

a. Despite the growing exposure to social media, OSY continue to consume information from different traditional media sources such as television, radio, newspapers, and movies, which OSY report continue to influence their behaviors.

b. Facebook is still the digital media application of choice among OSY who were the study's key informants, particularly for videos watched on mobile phones.

Media personalities and influencers play an important role in influencing OSY behaviors. Most key influencers mentioned by OSY who participated in this study are Filipino and Korean entertainment personalities (see Box 2, next page). Only one key influencer mentioned was a local politician.

c. Parents and parent-surrogates are also considered by OSY as key influencers in their lives. OSY appreciate their interactions with parents and surrogate parents, particularly for seeking guidance, advice on how to conduct themselves, and support in times of difficulty. These parental influences are particularly appreciated because of the sacrifices they have made for OSY and their siblings.

Even when everybody puts her down, she always comes back up. My mom's fatherin-law-my dad's dad-really doesn't like her. He always fights with my mom. Even my father's siblings argue with her. But my mom really endured all of that for us. She raised us all by herself in the 


\section{BOX 2 CELEBRITY AND MEDIA INFLUENCERS FOR OSY IN THE PHILIPPINES}

Media personalities and celebrities were consistently mentioned by OSY as key influences in their lives, including $\mathrm{RH}$ and FP behaviors. Among females, key influencers mentioned by both younger and older OSY were local female movie stars and television personalities such as Marian Rivera, Anne Curtis, Andie Eigenmann, Andrea Brilliantes, Angel Locsin, Liza Soberano, Rhian Ramos, Pia Wurtzbach, Korean actress Kim Go-Eun, and television host Vice Ganda. Female OSY said they admire these women because of their beauty, the strength of the characters they portray onscreen, and the kind of lives they live off screen, which female OSY aspire to emulate. Female respondents appreciated, in particular, key influencers who did not appear too liberated, as well as those who maintain their youthful looks even after having two or more children.

Among males, both younger and older, key influencers included personalities such as Dingdong Dantes, Enrique Gil, Vice Ganda, Dolphy, John Lloyd Cruz, Ronnie Alonte, and Coco Martin, as was Korean actor Park Bo-Gum. Rappers such as Waiian were also mentioned. Yul Servo, a local congressman, was also mentioned as a key influencer by one unmarried male youth respondent. Unlike female respondents, male youths, however, did not clearly articulate what about these personalities influenced their lives.

\section{five years that my dad was in prison. Five years. She had six children, but she did everything she could for all of us.}

-IDI, single female, Davao

\section{Conclusions}

\section{Social media and AYRH}

These study findings identify social media as the primary and most preferred source of RH and FP information, specifically for the four priority behaviors among OSY due to its accessibility and anonymity. This study is among the first in-depth study in the Philippines to explore social media use and SBC specifically related to OSY FP and RH behaviors. A number of specific findings relating to social media merit particular attention, especially for potential mutual shaping between social media and AYRH practices:

a. The acquisition of information by OSY from social media such as Facebook or YouTube suggest that OSY acquire $\mathrm{RH}$ and FP information both through active searching and passively. The information they receive is often inadequate, misleading, and inaccurate.

b. Social media's overall desirability as the preferred channel for SBC information and messages among OSY is largely attributable to its accessibility and anonymity. Both characteristics are essential for social media to remain a key preferred channel for OSY.

c. OSY are introduced to influencers who talk about issues relevant to them (e.g., failed romantic relationships, depression) through social media. While most OSY influencers are mainstream, or well-known through traditional media (e.g., Marian Rivera, Andi Eigenmann), some are alternative, or prominent in social media (e.g., Geo Ong). Influencers will remain a key channel through which messages on positive $\mathrm{RH}$ and FP behaviors can be shared with OSY and youth in general.

d. Besides positive influences of social media, it also remains the primary medium that facilitates the initiation of most risky behaviors (e.g., Facebook chat groups, private groups) and enables them to be learned, sustained, and propagated.

A well-designed and coordinated communication network with culture-centered and appropriate messages for OSY $\mathrm{RH}$ and FP are essential for relevant, beneficial, and effective SBC efforts to facilitate adoption of positive RH and FP behaviors among OSY in the Philippines.

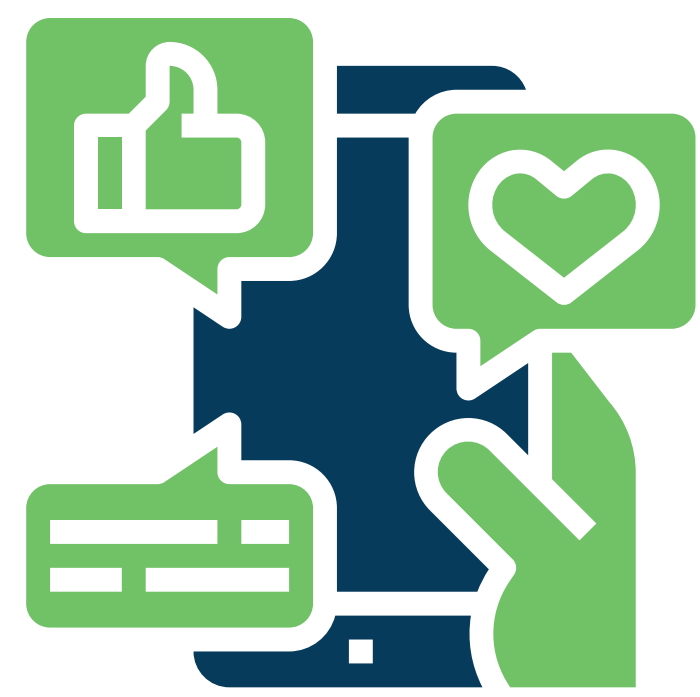




\section{Recommendations}

Based on the findings of the study, recommendations for strategic communication, policy, and service delivery include:

\section{Strategic communication}

- Given the fragmented nature of communication channels and sources for AYRH in the Philippines, design, build, implement, and formalize an SBC communications network of NGOs, faith-based organizations, government bodies or departments, civil society organizations, and individuals including parents, healthcare providers and adolescent champions themselves, engaged in SBC communications to improve $\mathrm{RH}$ and FP outcomes for OSY, particularly in urban areas. A well-designed and coordinated communications infrastructure with OSY-relevant messaging can help reach the most vulnerable and hard-to-reach OSY.

- Deploy social media applications for SBC that deliver RH and FP information that prioritize anonymity and confidentiality, the desired characteristics noted by youth in the study for preferred channels, while also providing accurate $\mathrm{RH}$ and FP information.

- Identify and work with key influencers who can be role models for adolescents and youth in the Philippines on positive AYRH behaviors. Identify gender responsive role models to reach male and female youth in the most effective ways.

- Identify and partner with influential and trusted institutions in complementary collaborations with SBC campaigns and messages disseminated to a broad range of OSY. Consider partnerships with traditional media including television broadcast networks and newspapers, given they continue to be relied on by OSY.

- Design messages that recognize culture as a central theme in how RH and FP behaviors are shaped as part of a formal "strategic RH, FP, and general health communication plan" targeting OSY. Frame messages as questions to motivate favorable behavior change in addition to encouraging further information-seeking on positive RH and FP behaviors.

- Pair loss-oriented messaging (e.g., "What will happen to me? How will it affect my future?") with messages promoting self-efficacy (e.g., "How can I protect myself?") and calls to action (e.g., "Talk to you parents about your $\mathrm{RH}$ and FP needs).
- Use visuals and relatable examples to help OSY distinguish and appreciate positive RH and FP behaviors and the potential negative consequences of risky behaviors.

- Systematically explore the role of social media, and Facebook in particular, to understand emerging risks confronting OSY via the medium, in particular for risky behaviors. Advance the understanding of protective and risk factors for $\mathrm{RH}$ and FP behaviors associated with social media platforms OSY use.

- Initiate dialogue and collaborate with Facebook and other social media companies to establish constructive discussions for providing both their data and digital platforms to enable the protection of young people actively engaged with their platforms.

\section{Research}

- Conduct multidisciplinary studies on communication contexts (including social and cultural factors) and integrating message strategies that are culturally-centered that can improve OSY RH and FP outcomes. Comprehensively map information flows related to OSY and AYRH (peer to peer, parent to child, media to OSY).

- Utilize human-centered design approaches with OSY to address perceived barriers in health communication initiated within various areas or levels both determined by and designed for OSY.

- Assess the cost-effectiveness of SBC messaging related to AYRH across different channels to identify cost-effective channels with the greatest potential for reach and impact.

- Test the efficacy of loss-oriented messages against positively framed messages targeted toward OSY on AYRH and related behaviors.

\section{Policy intervention}

- Review the implementation of existing laws and policies relevant to OSY RH and FP (e.g., Solo Parent Act, DoH Administrative Order 2013-0013-National Policy on Strategic Framework on Adolescent Health and Development, anchored on Universal Health Care) and ensure OSY issues are prioritized.

- Ensure implementation of RPRH (Responsible Parenthood and Reproductive Health) Act of 2012 within communities and among individuals through projects and initiatives that reach OSY and adolescents directly (e.g., under Gabayan ang Batang Ina [Guide the Teenage Mom] on Wheels, a group of 10 to 15 pregnant teenage girls brought to a city hospital for health checkups). 


\section{References}

1. Mmari, K. \& I. Sabherwal. 2013. "A review of risk and protective factors for adolescent sexual and reproductive health in developing countries: an update," Journal of Adolescent Health 53(5): 562-572. doi: 10.1016/j.jadohealth.2013.07.018

2. Tanaka, Y. 2017. "Knowledge, behavior and attitudes concerning STI prevention among out-of-school youth in the Philippines," Universal Journal of Public Health 5(3): 127-134. doi:10.13189/ ujph.2017.050307

3. World Health Organization (WHO). 2010. "The sexual and reproductive health of young adolescents in developing countries: Reviewing the evidence, identifying research gaps, and moving the agenda: report of a WHO technical consultation, Geneva, 4-5 November 2010." Geneva: WHO. Retrieved from: http://www. who.int/immunization/hpv/target/the_sexual_and_reproduc tive_health_of_young_adolescents_who_2011.pdf

4. Kirby, D. 2002. "The impact of schools and school programs upon adolescent sexual behavior," Journal of Sex Research 39(1): 27-33. doi:10.1080/00224490209552116

5. Hindin, M. and A. Fatusi, A. 2009. "Adolescent sexual and reproductive health in developing countries: an overview of trends and interventions," International Perspectives on Sexual and Reproductive Health 35(2): 58-62.

6. United Nations and United Nations Development Programme. 2016. "The joint programme on acceleration efforts to attain targets of Millennium Development Goal (MDG) 5 and Neonatal Component of MDG 4 thru joint programming: Rapid reduction of maternal and neonatal mortality in the Philippines (JPMNH)." Retrieved from http://mptf.undp.org/factsheet/fund/JPHOO.

7. Demographic Research and Development Foundation (DRDF) and University of the Philippines Population Institute (UPPI). 2014. "2013 YAFS4 key findings." Quezon City: DRDF and UPPI.

8. Kyilleh, J., P. Tabong, and B. Konlaan. 2018. "Adolescents' reproductive health knowledge, choices and factors affecting reproductive health choices: a qualitative study in the West Gonja district in Northern Region, Ghana," BMC International 18(1): 6. doi:10.1186/s12914-018-0147-5

9. World Bank. 2003. "Out-of-school children and youth in the Philippines: issues and opportunities," Human Development Sector Unit Report No. 23132-PH. Retrieved from https://openknowledge. worldbank.org/bitstream/handle/10986/14406/231320PHOwhite1portunities01public1.pdf

10. Albert, J. et al. 2012. "Profile of out-of-school children in the Philippines," Discussion paper series no. 2012-01. Makati City, Philippines: Philippine Institute for Development Studies. Retrieved from https://dirp3.pids.gov.ph/ris/dps/pidsdps1201.pdf

11. United Nations Population Fund \& Population Council. 2003. "The adolescent experience in-depth: using data to identify and reach the most vulnerable young people." New York: Population Council. Retrieved from https://www.popcouncil.org/uploads/pdfs/PGY AdolDataGuides/Philippines2003.pdf

\section{Contributors}

De La Salle UniversitySocial Development Research Center Ma. Jesusa Marco (Late) Maria Caridad Tarroja Jan Michael Alexandre Bernadas Ma. Angeles G. Lapena

\section{Acknowledgments}

We are grateful to the DLSU-SDRC and its research team, including the data collection team who conducted interviews and focusgroup discussions as part of the study, and the SDRC administrative team that coordinated the study on the ground in the Philippines. Thanks also go to USAID/Philippines and the USAID ReachHealth project for providing valuable feedback on the results. Most importantly, we are most grateful to the young people from Manila, Quezon City, Cebu and Davao, their parents and guardians and stakeholders who gave us their valuable time to provide important insights into the lives of young people in the Philippines.

We dedicate this brief in memory of Dr. Ma. Jesusa Marco, the Principal Investigator of this study, who passed away on 14 December 2019.

\section{Suggested citation:}

Breakthrough RESEARCH and De La Salle University. 2020. "Improving reproductive health and family planning outcomes among outof-school adolescents and youth in the Philippines," Programmatic Research Brief. Washington DC and Manila, Philippines: De La Salle University and Population Council.

(C)2020 The Population Council. All rights reserved.
Email

\section{BreakthroughResearch@popcouncil.org}

\section{Breakthrough RESEARCH | Population Council}

4301 Connecticut Ave., NW, Suite 280 | Washington, DC 20008

+12022379400 | breakthroughactionandresearch.org

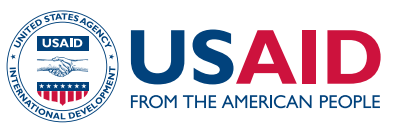

Breakthrough RESEARCH is made possible by the generous support of the American people through the United States Agency for International Development (USAID) under the terms of cooperative agreement no. AIDOAA-A-17-00018. The contents of this document are the sole responsibility of Breakthrough RESEARCH and Population Council and do not necessarily reflect the views of USAID or the United States Government.

\section{POPULATION} COUNCIL

Ideas. Evidence. Impact.

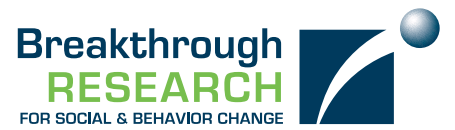

Breakthrough RESEARCH catalyzes social and behavior change (SBC) by conducting state-ofthe-art research and evaluation and promoting evidence-based solutions to improve health and development programs around the world. Breakthrough RESEARCH is a consortium led by the Population Council in partnership with Avenir Health, ideas42, Institute for Reproductive Health at Georgetown University, Population Reference Bureau, and Tulane University. 